\title{
Association of Severe Inflammatory Polyarthritis in Primary Sjögren's Syndrome: Clinical, Serologic, and HLA Analysis
}

\author{
KHULOOD MOHAMMED, JANET POPE, NICOLE LE RICHE, WILLIAM BRINTNELL, EWA CAIRNS, \\ ROBERT COLES, and DAVID A. BELL
}

\begin{abstract}
Objective. To evaluate the clinical, serologic, and MHC class II antigen characteristics of a group of patients with primary Sjögren's syndrome (SS) and severe arthritis.

Methods. A case-control study comparing 35 patients with primary SS: 17 with inflammatory arthritis, 18 without arthritis.

Results. All patients fulfilled criteria for primary SS. There were no demographic or clinical features other than inflammatory arthritis, often erosive, that distinguished patients with arthritis from those without. All patients had anti-Ro/SSA autoantibodies, most had anti-La/SSB autoantibodies, and a high percentage of these patients had anti-citrullinated peptide antibodies absent in those without inflammatory arthritis. HLA typing revealed that most patients with anti-citrulline antibodies expressed MHC class II molecules with the shared epitope (SE). The presence of DRB $1 * 0301$ linked to the expression of anti-Ro/SSA autoantibodies did not influence the level or frequency of anti-citrulline antibodies in these patients.

Conclusion. Severe arthritis with features resembling rheumatoid arthritis including erosive disease can occur in primary SS, particularly among those with anti-citrulline antibodies and the SE. (First Release June 1 2009; J Rheumatol 2009;36:1937-42; doi:10.3899/jrheum.080234)
\end{abstract}

Key Indexing Terms:

SJÖGREN'S SYNDROME INFLAMMATORY ARTHRITIS ANTI-CITRULLINE ANTIBODY

The clinical manifestations of primary Sjögren's syndrome (SS) in addition to sicca symptoms may be quite variable. Immuno-serologically, SS is usually characterized by polyclonal B cell activation with hypergammaglobulinemia and certain serum autoantibodies, particularly rheumatoid factor (RF) and anti-Ro/SSA and/or anti-La/SSB antibodies. Inflammatory polyarthritis is one of the clinically recog-

From the Department of Medicine, Division of Rheumatology, St. Joseph's Health Care London and University of Western Ontario; Department of Microbiology and Immunology, University of Western Ontario; and the Immunogenetics and Transplantation Laboratory, London Health Sciences Centre - University Campus, London, Ontario, Canada.

Supported by a grant from The Arthritis Society. Prof. Cairns is supported by an award from the Calder Foundation.

K. Mohammed, MD, Rheumatology Fellow, Division of Rheumatology, St. Joseph's Health Care London; J. Pope, MD, Professor; N. le Riche, MD, Professor, Department of Medicine, Division of Rheumatology;

W. Brintnell, PhD, Post-doctoral Associate, Department of Medicine and Microbiology and Immunology; E. Cairns, PhD, Professor, Department of Medicine, Division of Rheumatology, and Department of Microbiology and Immunology, University of Western Ontario; R. Coles, BSc, Medical Laboratory Technologist, London Health Sciences Centre; D.A. Bell, MD, Professor, Department of Medicine, Division of Rheumatology, University of Western Ontario.

Address reprint requests to Dr. D.A. Bell, Division of Rheumatology, Arthritis Centre, Monsignor Roney Building, St. Joseph's Health Care London, 268 Grosvenor Street, London, Ontario N6A 4V2, Canada.

E-mail: david.bell@sjhc.london.on.ca

Accepted for publication March 19, 2009. nized conditions that may exist in primary SS and when sicca symptoms are recognized in some patients with rheumatoid arthritis (RA), this is referred to as secondary SS. This may lead to confusing nomenclature since, while the arthropathy of primary SS is considered to be mild and nondestructive, patients with SS frequently have positive RF and hence may be initially diagnosed with RA.

We have recently observed a number of patients with clinical and serological features of primary SS who had a more flagrant polyarthritis, usually requiring the use of disease modifying antirheumatic drugs (DMARD), and who were often found to have erosive disease characteristic of RA. Many of these patients in addition to having serum RF also had elevated anti-citrullinated peptide antibodies (ACA; measured by $\mathrm{CCP}_{2}$ ), a serologic marker considered highly specific for RA.

Our objective was to document the clinical and serologic features of these patients with primary SS and severe polyarthritis and compare them to patients in our center who had a diagnosis of primary SS without inflammatory arthritis. We present the results of MHC class II typing in these patients, since the expression of certain MHC class II molecules is closely linked to expression of anti-citrulline antibodies (MHC class II molecules with the shared epitope) or anti-Ro/SSA and anti-La/SSB (MHC class II DRB1-0301) autoantibodies.

Personal non-commercial use only. The Journal of Rheumatology Copyright @ C 2009. All rights reserved. 


\section{MATERIALS AND METHODS}

Patients. All patients in this study $(\mathrm{n}=35)$ were seen in the ambulatory rheumatology clinic of an academic health sciences center. The 17 patients with primary SS and polyarthritis were primarily derived from the clinical practice of one author (DAB). Eighteen additional patients with primary SS and no arthritis were randomly recruited from the practices of other rheumatologists in this center, in order to determine if these patients exhibited significant differences other than arthritis compared to patients with primary SS and arthritis. All patients fulfilled diagnostic criteria for primary SS according to the American-European Consensus Group ${ }^{1}$. All patients in the study had sicca symptoms and a positive Schirmer's test and anti-Ro/SSA autoantibodies. In addition, 18 patients with RA were randomly selected from the clinical files of one author (DAB). All patients fulfilled American College of Rheumatology (ACR) criteria for RA ${ }^{2}$.

Laboratory testing. Anti-Ro/SSA, anti-La/SSB. These were detected by a commercial ELISA (Euroimmun Medical Diagnostics, Mississauga, Ontario, Canada). All the positive results were confirmed by counterimmunoelectrophoresis using purified antigens obtained from bovine spleen and thymus for Ro/SSA and calf and rabbit thymus for La/SSB.

Rheumatoid factor. IgM RF was determined by nephelometry and was considered positive at $>20 \mathrm{IU} / \mathrm{ml}$.

Anti-cyclic citrullinated peptide (anti-CCP) antibodies. Serum $\operatorname{IgG}$ anti-CCP antibodies were measured using a $\mathrm{CCP}_{2}$ ELISA (Euroimmun Medical Diagnostics). Levels of $\mathrm{IgG}$ anti- $\mathrm{CCP}_{2}>5 \mathrm{units} / \mathrm{ml}$ are considered positive in this assay.

HLA typing. HLA typing for the MHC class II B*1, DQB1, and DRB1*3, 4, 5 alleles was performed using polymerase chain reaction sequence-specific priming (PCR-SSP) utilizing commercial typing kits (QIAgen, Mississauga, Ontario, Canada). Amplification was performed using the following cycling characteristics: initial melt $94^{\circ} \mathrm{C}$ for 1 min followed by 10 cycles of $94^{\circ} \mathrm{C} 10$ $\mathrm{s}, 65^{\circ} \mathrm{C} 60 \mathrm{~s}$, followed by 20 cycles of $94^{\circ} \mathrm{C} 10 \mathrm{~s}, 61^{\circ} \mathrm{C} 50 \mathrm{~s}, 72^{\circ} \mathrm{C} 30 \mathrm{~s}$. Amplified DNA was electrophoresed on $2 \%$ agarose gel and stained with ethidium bromide, and the resulting pattern was photographed. Analysis of gel patterns was performed with the Helmberg SCORE ${ }^{\mathrm{TM}}$ computer program (QIAgen) to give generic and allele-level typing using the World Health Organization nomenclature system for HLA ${ }^{3}$

Radiographic measurements. Standard radiographs of the hands and feet were taken from all patients at the time of anti-CCP analysis and were evaluated for the presence of erosions by one reviewer.

Statistical analysis. Student's t test was used for testing the significance of differences between the 2 groups of patients. All tests were 2-sided, and probability (p) values $<0.05$ were considered significant. In some comparisons, chi-square analysis was performed to compare differences between groups.

\section{RESULTS}

The SS patients with arthritis presented with a symmetrical inflammatory polyarthritis that targeted the wrists, metacarpophalangeal (MCP), proximal interphalangeal, and metatarsophalangeal (MTP) joints most frequently. Characteristically, there was swelling in small joints of hands, wrists, and feet accompanied by tenderness without deformity before treatment. Some patients had signs of synovitis in the knees and ankles (swelling and tenderness). Deformities were not usually seen (e.g., joint subluxation, ulnar deviation at MCP joints). None had detectable rheumatoid nodules. Clinical and laboratory features of patients in the arthritis group $(\mathrm{n}=17)$ and nonarthritis group $(\mathrm{n}=18)$ are compared in Table 1 and Table 2. There was no significant difference in the age, sex, or duration of disease
Table 1. Baseline characteristics of the subjects with SS

\begin{tabular}{lcc}
\hline Characteristic & $\begin{array}{c}\text { Arthritis } \\
\text { Group }\end{array}$ & $\begin{array}{c}\text { Non-Arthritis } \\
\text { Group }\end{array}$ \\
\hline No. of subjects (n) & 17 & 18 \\
Age, yrs, range (mean) & $27-85(56)$ & $25-81(53)$ \\
Sex, F/M & $16 / 1$ & $16 / 2$ \\
Disease duration, yrs, range (mean) & $2-28(15)$ & $2-31(16)$ \\
Systemic features, \% (n) & & \\
Severe polyarthritis & $100(17 / 17)$ & 0 \\
Sicca symptoms & $100(17 / 17)$ & $100(18 / 18)$ \\
Rash & $29(5 / 17)$ & $39(7 / 18)$ \\
Raynaud's & $35(6 / 17)$ & $33(6 / 18)$ \\
Peripheral neuropathy & $23.5(4 / 17)$ & $16(3 / 18)$ \\
Autoimmune thyroiditis & $11.7(2 / 17)$ & $11(2 / 18)$ \\
DMARD use & $78(13 / 17)^{*}$ & $11(2 / 18)^{* *}$ \\
\hline
\end{tabular}

* Either methotrexate or leflunomide in Subjects 1-7 and 17, etanercept in Subject 4, and others received hydroxychloroquine (see Table 3). ** Hydroxychloroquine used for arthralgias and fatigue.

Table 2. Laboratory and serological features of the subjects with SS.

\begin{tabular}{lcc}
\hline Feature & $\begin{array}{c}\text { Arthritis } \\
\text { Group (\%) } \\
\mathrm{n}=17\end{array}$ & $\begin{array}{c}\text { Non-Arthritis } \\
\text { Group (\%) } \\
\mathrm{n}=18\end{array}$ \\
\hline Anemia & $6(35)$ & $5(28)$ \\
Cytopenias & $9(53)$ & $10(55.5)$ \\
Elevated IgG & $11(65)$ & $9(50)$ \\
FANA-positive & $4(23.5)$ & $5(28)$ \\
Rheumatoid factor-positive & $16(94)$ & $15(83)$ \\
Anti-Ro/SSA-positive & $17(100)$ & $18(100)$ \\
Anti-Ro/SSA plus anti-La/SSB & $9(52)$ & $11(61)$ \\
Anti-citrullinated peptide antibodies & $8(47)$ & 0 \\
Presence of erosions & $9(53)$ & 0 \\
\hline
\end{tabular}

FANA: fluorescent antinuclear antibody.

(mean $15.5 \mathrm{yrs}$ ) between those with and without arthritis. Among the additional clinical features seen in both groups of patients with primary SS were erythematous maculopapular photosensitive rashes (usually in the forearms and neck and occasionally the face), which were reported in $29 \%-39 \%$ of patients, symptoms of Raynaud's phenomenon without cyanosis or signs of peripheral vasculitis (33\%-35\%), and mild peripheral neuropathy (usually sensory, glove or stocking distribution) in 16\%-23\%. Obvious parotid or lacrimal gland swelling was usually not present. None of the extraarticular features of SS distinguished the arthritis from the nonarthritis group.

DMARD use was restricted to the arthritis group (78\%), but some patients without arthritis were receiving antimalarials for systemic/constitutional symptoms and/or rashes. None of the nonarthritis patients were receiving DMARD or immunosuppressive drugs. Current DMARD use in the arthritis group consisted of methotrexate (7/17), hydroxychloroquine (4/17), leflunomide (1/17), and the biologic therapy etanercept (1/17). 
There was no difference in the frequency of most laboratory serologic tests in the 2 groups (Table 2). Notably, RF occurred with a similar frequency in both groups, and all patients had anti-Ro/SSA antibody. Among the 35 patients with SS, 13 (37\%) had anti-Ro/SSA antibody only, and the remaining $63 \%$ had both anti-Ro/SSA and anti-La/SSB. None had anti-DNA or anti-Sm or anti-nRNP antibodies. Polyclonal hypergammaglobulinemia was common but was present at a similar frequency in both groups of patients. Among the arthritis group of patients, equal numbers had anti-Ro/SSA only or anti-Ro/SSA and anti-La/SSB. Among the nonarthritis group, slightly more patients $(70 \%)$ had both anti-Ro/SSA and anti-La/SSB. This difference was not significant.

No additional clinical features distinguished these SS patients with anti-Ro/SSA alone compared to those with both anti-Ro/SSA and anti-La/SSB (data not shown). We compared the clinical, demographic, and serologic features of the patients with SS to 18 patients with RA chosen at random from our files who fulfilled ACR criteria for RA. These patients had demographic features similar to the patients who had SS in terms of age, sex, and disease duration. None of these patients had clinical features, either symptoms or signs, of SS. Most of the patients were RF-positive (82\%) and had typical erosive disease targeting the small joints of hands, wrists, and feet $(91 \%)$. None of the patients had anti-Ro/SSA or anti-La/SSB autoantibodies using the same assay as those tested among patients with SS.

All patients with arthritis had plain radiographs of the wrists, MCP, or MTP joints. Among the patients with SS, radiographic changes were present in 9/17 (53\%), consisting of classical erosive changes typical and indistinguishable from RA. Sixteen (83\%) of the 18 RA patients selected for comparison had radiographic erosions typical of RA involving the small joints of the hands, wrists, and feet.

Anti-cyclic citrullinated antibodies (ACA). ACA were measured by binding to $\mathrm{CCP}_{2}$ and were exclusive to patients with primary SS with arthritis and the patients with RA. Eight (47\%) of 17 had such antibodies (Table 2). Concentrations of serum ACA among these patients are shown in Table 3. The clinical features of ACA-positive and ACA-negative patients are compared in Table 4. Apart from the synovitis exclusive to the ACA-positive patients, no other clinical or serologic features distinguished these 2 groups of patients with primary SS. Among the 8 arthritis patients, $6(75 \%)$ had erosions (Table 4). ACA were present in most (82\%) of the patients selected for comparison purposes with RA.

HLA typing. Details of the DRB1* alleles of the patients with SS are shown in Table 3. Table 5 provides a further analysis of these data and compares certain of the DRB1 alleles in the arthritis and the nonarthritis group. Among the arthritis group, DRB1 alleles with the shared epitope (SE) were present in 7/17 (41\%) patients, and one (Subject 3) was homozygous for DRB1* alleles with the SE (Table 3).
DRB1* alleles with the SE were present in only $2 / 18$ patients in the nonarthritis group. DRB $1 * 0301$, known to be increased among anti-Ro/SSA patients with $\mathrm{SS}^{4}$, was detected in 22 of 35 patients. However, this allele was present at a similar frequency in patients with arthritis (9/17) and without arthritis (13/18).

Further analysis of the relationship between these HLA alleles and presence of anti-Ro/SSA and anti-La/SSB antibodies in those with and without arthritis was performed. Most patients (82\%) with anti-Ro/SSA antibody alone expressed the HLA-DRB1 allele 0301; 33\% of those with both anti-Ro/SSA and anti-La/SSB expressed only the DRB1 allele 0301; 50\% of patients carried both DRB1*0301 and DRB1*1501.

We also analyzed the frequency of these DRB1 alleles among the arthritis and nonarthritis patients. Among the arthritis patients with anti-Ro/SSA antibodies alone, 50\% expressed the DRB1 allele 0301 and only 15\% expressed both DRB1*0301 and DRB1*1501. Among those with both anti-Ro/SSA and anti-La/SSB, most (66\%) expressed only the DRB1 allele 0301. Among the nonarthritis group, most (70\%) had anti-Ro/SSA and anti-La/SSB antibody and most of these expressed HLA-DRB $1 * 0301$ and 1501 .

We compared the frequency of DRB1 alleles among patients with and without ACA (Table 6). The SE was significantly more common in patients with ACA. In addition, $7 / 16$ patients expressed the DRB $1 * 1501$ allele, which has been linked to $\mathrm{SS}^{5}$. We have shown previously that soluble DRB1* alleles with the 1501 specificity bind citrullinated peptide with a very high affinity similar to soluble SE alleles ${ }^{6}$. The DRB1*1501 allele was also significantly more common in this group of patients with SS who had ACA. Among the 18 patients with RA, 11 were typed for HLA: $7 / 11$ expressed the shared epitope. Five of these 7 had ACA. Four coexpressed the HLA allele 0301, and 2 of these also had ACA.

Finally, we analyzed our data to determine if the presence of the DRB $1 * 0301$ allele influenced either the frequency or concentration of serum ACA, as reported ${ }^{7}$. Table 7 shows that this allele had no significant effect on ACA in this group of patients with SS.

\section{DISCUSSION}

We investigated the clinical features and MHC class II and serologic expression of ACA in a group of patients who had primary SS (all with anti-Ro/SSA antibodies) with and without severe inflammatory polyarthritis. Our data describe a subset of SS patients who had clinical and serologic features typical of primary SS but who also had severe inflammatory polyarthritis resembling RA, frequently requiring treatment with DMARD. Among a similar group of patients with established RA, most of whom had ACA and erosive changes on radiographs, none had anti-Ro/SSA or anti$\mathrm{La} / \mathrm{SSB}$ autoantibodies.

Personal non-commercial use only. The Journal of Rheumatology Copyright @ @ 2009. All rights reserved. 
Table 3. HLA-DRß1 alleles in Sjögren's syndrome. Alleles printed in bold type indicate occurrence of the shared epitope.

\begin{tabular}{|c|c|c|c|c|c|}
\hline \multicolumn{3}{|c|}{ Arthritis Group, $\mathrm{n}=17$} & \multicolumn{3}{|c|}{ Non-Arthritis Group, $\mathrm{n}=18$} \\
\hline Subject & DRB1* Alleles & $\mathrm{ACA}^{\dagger}$ & Subject & DRB $1 *$ Alleles & ACA \\
\hline 1 & 0401, 1401 & $+(15)$ & 1 & 0301, 0801 & - \\
\hline 2 & 0301,1101 & $+(>100)$ & 2 & 1501,1001 & - \\
\hline 3 & 0401,0404 & - & 3 & 1501,0301 & - \\
\hline 4 & 0301,0401 & $+(100)$ & 4 & 0701,1101 & - \\
\hline 5 & 1501,0303 & $+(100)$ & 5 & 0301, 0801 & - \\
\hline 6 & 0301, 0701 & - & 6 & 1501,1301 & - \\
\hline 7 & 0301,0407 & - & 7 & 1501,0301 & - \\
\hline 8 & 1501, 0401 & $+(72)$ & 8 & 1501,0301 & - \\
\hline 9 & 0101, 0301 & $+(100)$ & 9 & 1501,1101 & - \\
\hline 10 & 0801,1501 & - & 10 & 1501,0301 & - \\
\hline 11 & 0301,1501 & $+(100)$ & 11 & 1601,0301 & - \\
\hline 12 & $\mathbf{0 4 0 4}, 0901$ & - & 12 & 0401, 1302 & - \\
\hline 13 & 0301,1101 & - & 13 & 0301,1302 & - \\
\hline 14 & 0301,1601 & - & 14 & 1501,0301 & - \\
\hline 15 & 0101, 1502 & $+(29)$ & 15 & 0301, 0701 & - \\
\hline 16 & 0301, 0801 & - & 16 & 0301,1601 & - \\
\hline \multirow[t]{2}{*}{17} & 1301,0801 & - & 17 & 0301,1302 & - \\
\hline & & & 18 & 0301,1501 & - \\
\hline
\end{tabular}

$\dagger$ Units/ml of anti-citrulline antibody.

Table 4. Clinical and serological features of SS patients with and without anti-citrulline antibodies.

\begin{tabular}{lcc}
\hline Feature & $\begin{array}{c}\text { ACA-positive, } \\
\mathrm{n}=8\end{array}$ & $\begin{array}{c}\text { ACA-negative, } \\
\mathrm{n}=27\end{array}$ \\
\hline Age, yrs, range (mean) & $22-83(53)$ & $27-82(54)$ \\
Sex, F/M & $7 / 1$ & $24 / 3$ \\
Disease duration, yrs, range (mean) & $2-27(14)$ & $2-29(15)$ \\
Synovitis, \% & 100 & 0 \\
Radiographic erosions (\%) & $6 / 8(75)$ & $3 / 27(11)$ \\
Extraglandular involvement (\%) & $7 / 8(87)$ & $19 / 27(70)$ \\
Rheumatoid factor-positive (\%) & $8 / 8(100)$ & $22 / 27(81)$ \\
Anti-Ro/SSA-positive (\%) & $8 / 8(100)$ & $27 / 27(100)$ \\
\hline
\end{tabular}

Table 5. Summary of HLA-DRß1 alleles in patients with SS.

\begin{tabular}{lccc}
\hline Allele & $\begin{array}{c}\text { Arthritis Group, } \\
\mathrm{n}=17(\%)\end{array}$ & $\begin{array}{c}\text { Non-Arthritis Group, } \\
\mathrm{n}=18(\%)\end{array}$ & $\mathrm{p}$ \\
\hline HLA-DRB1* & & & \\
0301 & $9 / 17(53)$ & $13 / 18(72)$ & $\mathrm{NS}^{\dagger}$ \\
1501 & $4 / 17(23)$ & $9 / 18(50)$ & $\mathrm{NS}$ \\
SE & $7 / 17(41)$ & $2 / 18(11)$ & $\mathrm{NS}$ \\
SE or 1501 & $6 / 17(35)$ & $10 / 18(55)$ & $\mathrm{NS}$ \\
\hline
\end{tabular}

\footnotetext{
${ }^{\dagger}$ Not statistically significant $(\mathrm{p} \geq 0.05)$.
}

It is well recognized that arthritis can occur in primary SS. Bjerrum, et $a l^{8}$ reported that articular symptoms were more severe in patients with SS whose disease started at an earlier age, and others report that arthralgias are present only periodically ${ }^{9}$ and rarely result in joint deformity or ero$\operatorname{sion}^{10}$. We found no differences in the age or disease dura-
Table 6. Summary of anti-citrulline antibody and HLA-DRB1* alleles in patients with SS.

\begin{tabular}{lccc}
\hline DRB1* Alleles & $\begin{array}{c}\text { ACA-positive, } \\
\mathrm{n}=8(\%)\end{array}$ & $\begin{array}{c}\text { ACA-negative, } \\
\mathrm{n}=27(\%)\end{array}$ & $\mathrm{p}$ \\
\hline 0301 & $4 / 8(50)$ & $14 / 27(52)$ & $>0.05$ \\
1501 & $2 / 8(25)$ & $10 / 27(37)$ & $>0.05$ \\
$\mathrm{SE}$ & $5 / 8(62.5)$ & $4 / 27(15)$ & $>0.05$ \\
$\mathrm{SE}+1501$ & $7 / 8(87.5)$ & $4 / 27(15)$ & 0.0009 \\
\hline
\end{tabular}

Table 7. Influence of HLA-DRB1*0301 on frequency and level of anti-citrulline antibody.

\begin{tabular}{lccc}
\hline & $\begin{array}{c}\text { 0301-positive, } \\
\mathrm{n}=22\end{array}$ & $\begin{array}{c}\text { 0301-negative, } \\
\mathrm{n}=13\end{array}$ & $\mathrm{p}$ \\
\hline ACA-positive (\%) & $4 / 22(18)$ & $4 / 13(30)$ & $\mathrm{NS}^{\dagger}$ \\
$\begin{array}{c}\text { ACA level, units/ml, } \\
\text { mean (range) }\end{array}$ & $100(100)$ & $53(15-100)$ & $\mathrm{NS}$ \\
\hline $\mathrm{p}<0.05$. & & \\
\hline
\end{tabular}

tion in our group of patients with primary SS with arthritis compared to those without arthritis, and the arthritic symptoms were persistent and frequently characterized by erosions.

Mariette, et $a l^{11}$ reported that unlike patients with RA, those with primary SS with inflammatory arthritis responded poorly to treatment with anti-tumor necrosis factor- $\alpha$-blocking agents such as infliximab. This may suggest a different mechanism responsible for the arthritis in patients with RA and those with SS.

Gottenberg, et $a l^{12}$ have reported that among a group of 
134 patients with primary SS, $7.5 \%$ had ACA, only $20 \%$ of whom had arthritis. No HLA typing was reported in this study. However, they excluded patients with erosive arthritis from their analysis. In our study, all patients with ACA had arthritis, particularly those with erosive disease. We did not exclude these SS patients from the analysis because they had clinical and laboratory features more typical of SS than RA.

In previous studies in RA, it was found that ACA were strongly associated with HLA-DRB1* alleles with the SE amino acid consensus sequence ${ }^{13,14}$. We previously reported that citrullinated peptides bind with high affinity to soluble SE alleles and also to MHC class II molecules with the DRB1*1501 allele 5 . In our present study, we show that among the group of patients with arthritis, those with ACA were significantly more likely to express the SE than those lacking ACA. Only those SS patients with arthritis had ACA. As anticipated, the selected RA patients had a high frequency of ACA. We also found a strong association between the expression of the SE or non SE (DRB1*1501) and ACA in the arthritis group. Thus primary SS patients with arthritis compared to those without arthritis are more apt to express the SE ( $41 \%$ vs $11 \%$, respectively) and ACA (47\% vs $0 \%)$.

The relationship between clinical and serologic features and HLA antigens was investigated by Gottenberg and coworkers ${ }^{15}$ among a group of 149 patients with primary SS. Two major conclusions were that, first, only those patients with primary SS and anti-Ro/SSA and/or anti-La/SSB autoantibodies expressed a significant association with HLA antigens. Further, HLA-DRB*0301 was expressed in patients with both anti-Ro/SSA and anti-La/SSB, while HLA-DRB1*1501 was expressed only in those who had anti-Ro/SSA. Arthralgia or arthritis was equally represented in both groups of patients. An analysis of the relationship between the serologic features of our group in particular, anti-Ro/SSA and anti-La/SSB antibodies and the expression of DRB1 alleles did not show this kind of association. Thus, most patients with anti-Ro/SSA antibodies alone expressed HLA-DRB $1 * 0301$, while only $33 \%$ of those with both anti-Ro/SSA and anti-La/SSB expressed HLA-DRB $1 * 0301$ and half of these also expressed DRB $1 * 1501$.

Our studies highlight several important issues. First, as stated, it is clear that despite the impression that the arthritis that occurs in primary SS is mild and nondestructive, we found that in some patients this arthritis may share many of the clinical, serologic, and radiologic features of "RA." The alternative interpretation, that the arthritis patients we describe simply have RA with secondary "sicca syndrome," seems highly unlikely. First, these patients all had serum anti-Ro/SSA and half had anti-La/SSB antibodies, serologic features distinctly unusual in $\mathrm{RA}^{16}$. Second, these patients commonly had other systemic features unusual in RA (Table 1). Finally, a high proportion of these patients expressed the
HLA-DRB $1 * 0301$ allele relatively uncommon in RA but characteristic of patients with primary SS, particularly in those with anti-Ro/SSA antibody ${ }^{17}$. As stated, none of the RA patients we analyzed who had demographic features similar to the SS group had anti-Ro/SSA or anti-La/SSB autoantibody.

The unexpected occurrence of significant arthritis and of ACA in these patients with primary SS raises the question of the specificity of ACA for RA. It appears that ACA identify patients with primary SS who have or who may develop significant inflammatory polyarthritis. Many of these SS patients with arthritis expressed the SE (41\%), previously linked to the presence of RA. No other demographic, clinical, or serologic features appeared to distinguish these 2 groups of patients with primary SS.

Our findings raise the question about how RA itself is defined, since many of our study patients with primary SS and arthritis would fulfill ACR criteria for RA ${ }^{2}$. It has been acknowledged that these criteria are imperfect in defining what we understand as $\mathrm{RA}^{18}$. Significant inflammatory arthritis and ACA have also recently been reported in a minority of patients with other connective tissue diseases such as systemic lupus erythematosus (SLE) and scleroder$\mathrm{ma}^{19,20}$. A recent study of erosive arthritis among a group of patients with SLE in the UK, many of whom had ACA, highlights this issue ${ }^{21}$. Most of these patients with ACA also expressed HLA-DRB1 alleles with the SE. A possibility that needs to be considered is that ACA may be the phenotypic expression of genes expressing the SE that are associated with or identify inflammatory polyarthritis occurring either alone (as in "RA") or in association with other disorders such as SLE, SS, or scleroderma. We have recently shown experimentally that the immune response to citrulline can be readily achieved and cause arthritis in DR4-IE transgenic mice but not wild-type mice ${ }^{22}$. Moreover, specifically purified IgG ACA from humans with RA can induce arthritis in Fc $\gamma \mathrm{R}$ IIB-/- mice ${ }^{23}$. It will be of interest to determine whether ACA from these non-RA patients have the same arthritogenic property.

Finally, it has been reported ${ }^{7}$ that the DRB $1 * 0301$ allele may negatively influence the frequency or level of ACA among patients with RA who have this allele on one parental chromosome. Analysis of our data, admittedly from small numbers of patients, does not support this conclusion. Thus, those who express the DRB1* allele 0301 had a somewhat higher level of ACA, but not a significantly increased frequency of this antibody.

We describe a subset of patients with primary Sjögren's syndrome who had a severe inflammatory polyarthritis requiring DMARD treatment, many of whom had erosive disease. A number of these patients expressed ACA and had MHC class II genes, which are known to be associated with the expression of this antibody.

Personal non-commercial use only. The Journal of Rheumatology Copyright @ 2009. All rights reserved. 


\section{REFERENCES}

1. Vitali C, Bombardieri S, Jonsson R, et al. Classification criteria for Sjögren's syndrome: a revised version of the European criteria proposed by the American-European Consensus Group. Ann Rheum Dis 2002;61:554-58.

2. Arnett FC, Edworthy SM, Bloch DA, et al. The American Rheumatism Association 1987 revised criteria for the classification of rheumatoid arthritis. Arthritis Rheum 1988;31:315-24.

3. Robinson J, Waller MJ, Parham P, et al. IMGT/HLA and IMGT/MHC: sequence databases for the study of the major histocompatibility complex. Nucl Acids Res 2003;31:311-14.

4. Arnett FC, Bias WB, Reveill JD. Genetic studies in Sjögren's syndrome and systemic lupus erythematosus. J Autoimmunity 1989;2:403-13.

5. Hill JA, Sidney J, Sette A, et al. DRB $1 * 1501$ which does not contain the classic shared epitope sequence can bind citrullinated peptides with high affinity and supports anti-citrullinated peptide antibody (ACPA) production in rheumatoid arthritis patients [abstract]. Arthritis Rheum 2005;52 Suppl:S150.

6. Hill JA, Sidney J, Sette A, Jevnikar AM, Bell DA, Cairns E. Cutting edge: The conversion of arginine to citrulline allows for a high-affinity peptide interaction with the rheumatoid arthritis-associated HLA-DRB1*0404 MHC class II molecule. J Immunol 2003;171:538-41.

7. Verpoort KN, van Gaalen FA, van der Helm-van Mil AH, et al. Association of HLA DR3 with anti-cyclic citrullinated peptide antibody-negative rheumatoid arthritis. Arthritis Rheum 2005;52:3058-62.

8. Bjerrum K, Prause JU. Primary Sjögren's syndrome: a subjective description of the disease. Clin Exp Rheumatol 1990;8:283-8.

9. Pease CT, Shattles W, Barrett NK, Maini RN. The arthropathy of Sjögren's syndrome. Br J Rheumatol 1993;32:609-13.

10. Haga HJ, Rygh T, Jacobsen H, Johannessen AC, Mjanger O, Jonsson R. Sjögren's syndrome: new diagnostic aspects. Tidsskr Nor Laegeforen 1997;117:2197-200.

11. Mariette X, Ravaud P, Steinfeld S, et al. Inefficacy of infliximab in primary Sjögren's syndrome: results of the randomized, controlled Trial of Remicade in Primary Sjögren's Syndrome (TRIPSS). Arthritis Rheum 2004;50:1270-76.

12. Gottenberg JE, Mignot S, Nicaise-Rolland P, et al. Prevalence of anti-cyclic citrullinated peptide and anti-keratin antibodies in patients with primary Sjögren's syndrome. Ann Rheum Dis 2005;64:114-7.
13. Goldbach-Mansky R, Lee J, McCoy A, et al. Rheumatoid arthritis associated autoantibodies in patients with synovitis of recent onset. Arthritis Res 2000;2:236-43.

14. Huizinga TWJ, Amos CI, van der Helm-van Mil AH, et al. Refining the complex rheumatoid arthritis phenotype based on specificity of the HLA DRß1 shared epitope for antibodies to citrullinated proteins. Arthritis Rheum 2005;52:3433-8.

15. Gottenberg JE, Busson M, Loiseau P, et al. In primary Sjögren's syndrome, HLA class II is associated exclusively with autoantibody production and spreading of the autoimmune response. Arthritis Rheum 2003;48:2240-45.

16. Boire G, Ménard HA, Gendron M, Lussier A, Myhal D. Rheumatoid arthritis: anti-Ro antibodies define a non-HLA DR4 associated clinicoserological cluster. J Rheumatol 1993;20:1654-60.

17. Arnett FC, Hamilton RG, Reveille JD, Bias WV, Harley JB, Reichlin M. Genetic studies of Ro (SS-A) and La (SS-B) autoantibodies in families with systemic lupus erythematosus and primary Sjögren's syndrome. Arthritis Rheum 1989;32:413-9.

18. Symmons DPM, Hazes JMW, Silman AJ. Cases of early inflammatory polyarthritis should not be classified as having rheumatoid arthritis. J Rheumatol 2003;30:902-4.

19. Matsui T, Shimada K, Ozawa N, et al. Diagnostic utility of anti-cyclic citrullinated peptide antibodies for very early rheumatoid arthritis. J Rheumatol 2006;33:2390-7.

20. Ingegnoli F, Galbiati V, Zeni S, et al. Use of antibodies recognizing cyclic citrullinated peptide in the differential diagnosis of joint involvement in systemic sclerosis. Clin Rheumatol 2007;26:510-4.

21. Chan MT, Owen P, Dunphy J, et al. Associations of erosive arthritis with anti-cyclic citrullinated peptide antibodies and MHC class II alleles in systemic lupus erythematosus. J Rheumatol 2008;35:77-83.

22. Hill JA, Bell DA, Brintnell W, et al. Arthritis induced by post-translationally modified (citrullinated) fibrinogen in DR4-IE transgenic mice. J Exp Med 2008;205:967-79.

23. Li M, Brintnell W, Bell DA, Cairns E. The arthritogenicity of human anti-citrullinated peptide antibodies in rheumatoid arthritis [abstract]. Arthritis Rheum 2008;58 Suppl:S936. 